\title{
利用被动式采样膜评估水中腐殖酸对苍和䓛 生物有效性的影响
}

陈 珊 柯润辉 黄圣彪 孙立伟 查金苗 王子健“

(中国科学院生态环境研究中心环境水质学国家重点实验室, 北京 100085.* 联系人, E-mail: wangzj@rcees.ac.cn)

\begin{abstract}
摘要 以范和䓛两种多环芳烃(PAHs)为研究对象, 在实验室模拟条件下, 研究了水中不同含量的腐殖 酸(HA)(0 15 mg $\cdot \mathrm{L}^{-1}$, 以溶解性有机碳浓度表征)对 PAHs 在三油酸甘油酯-醋酸纤维素复合膜(TECAM) 中和日本青鲳鱼体内富集的影响, 比较了两种采样介质中 PAHs 表观富集速率常数随 HA 浓度变化的趋 势, 并利用模型拟合计算了两种方法得到的范和䓛的腐殖酸-水平衡分配常数 $\left(K_{\mathrm{DOC}}\right)$ 值. 结果表明, 水中 存在的 HA 对 TECAM 和青鲳富集两种 PAHs 有显著影响, 随着 HA 浓度的增大, PAHs 在 TECAM 膜中 和青鲳体内的富集浓度显著降低, 其表观富集速率常数也随 HA 浓度的增大而减小. 用 TECAM 萃取和 青鳞富集实验得到的两种 PAHs 的 $\lg K_{\mathrm{DOC}}$ 分别为 4.63 和 4.52(范), 以及 5.83 和 5.76(䓛); 两种方法所得 的 lg $K_{\mathrm{DOC}}$ 值相互之间有很好的可比性, 并与文献报道值相吻合. 研究结果证明, TECAM 能够选择性地 富集水相中自由溶解形态的 PAHs, 且富集行为与鱼体类似, 因此可作为一种生物模拟采样器来研究水 环境中存在的溶解性有机碳对 PAHs 生物有效性的影响.
\end{abstract}

\section{关键词 生物有效性 多环芳烃 腐殖酸 生物模拟采样 分配系数 溶解性有机碳}

天然水体中普遍含有各种类型的溶解性有机碳 (DOC), 其中大部分为腐殖酸类物质 ${ }^{[1]}$. 研究表明, DOC能吸附并结合疏水性有机物(HOCs), 从而在很 大程度上影响其在水体环境中的迁移、转化及归趋行 为 ${ }^{[2]}$. 一方面, 与DOC结合提高了HOCs在水中的表 观溶解度, 并促使其随DOC在水体中的流动而扩散 到其他地方; 另一方面, 结合态的HOCs体积和极性 变大, 难以透过生物膜在生物体内累积, 又会极大地 降低其在生物体内的富集浓度和生物有效性 ${ }^{[3]}$. 因此, 只有自由溶解的部分才能真实地反映出水体环境中 HOCs的实际毒性效应 ${ }^{[2]}$. 为了准确地进行污染物的 环境风险评价, 需要定量测定水体环境中的DOC对 污染物生物有效性的影响, 通常用污染物的溶解性 有机碳-水平衡分配常数 $\left(K_{\mathrm{DOC}}\right)$ 来表征. $K_{\mathrm{DOC}}$ 是描述有 机污染物在水相和DOC相间分配的重要参数, 它与 水体中的有机碳含量共同决定了 HOCs的自由溶解态 浓度, 所以可以通过测定污染物总浓度和 $K_{\mathrm{DOC}}$ 值来 准确计算出水体中的自由溶解态浓度. 目前常用的 $K_{\mathrm{DOC}}$ 测定方法有生物富集、平衡透析、苂光猝灭、反 相色谱、固相微萃取等 ${ }^{[3,4]}$. 多环芳烃类物质 $(\mathrm{PAHs})$ 是一类全球性污染物，具有潜在的“三致”效应，
可经由多种途径进入水体, 并在生物体的脂肪中累 积 ${ }^{[5]}$. 由于其典型的疏水性, 在探讨PAHs在水体环境 中的分布、归趋及其生物毒性时, 考虑DOC吸附因素 所造成的影响就显得尤为重要 ${ }^{[6,7]}$.

被动式采样的过程与亲脂性有机物透过生物膜 的扩散过程类似, 因此经常被用于模拟生物富集研 究. 自 Södergren ${ }^{[8]}$ 首次提出用正己烷透析带富集疏 水性有机物以来, 半渗透膜被动式采样器已被广泛 地应用于监测各种水体环境中痕量有机污染物的研 究中 ${ }^{[9,10]}$, 其中运用最广泛的是由美国地质调查局 Huckins等人 ${ }^{[11]}$ 发展的三明治构型的半渗透膜被动式 采样器(SPMD). 本实验室开发的三油酸甘油酯-醋酸 纤维素复合膜(TECAM)是一种新型的被动式采样器, 能够很好地富集有机氯农药、PAHs等疏水性有机物, 相对于其他采样方法, 具有成本低廉、制备和使用操 作简单、易于净化等优点 ${ }^{[9,12]}$. 但其能够选择性地富 集水体中自由溶解态有机物的特性尚未得到确证, 在评估实际水体中HOCs生物有效性方面的应用也有 待展开. 由此, 本研究尝试以微耗式采样(negligible depletion sampling $)^{[13]}$ 的方式利用TECAM测定不同浓 度的腐殖酸对膜富集两种多环芳烃范和䓛的影响, 通 
过表观富集速率常数与实际富集速率常数间的偏离 度来计算目标化合物在腐殖酸和水相间的分配系数 $K_{\mathrm{DOC}}$, 该值可用于估测水中两种PAHs的生物有效态 浓度, 从而定量研究水体环境中的DOC对两种PAHs 生物有效性的影响; 并与同样条件下暴露的日本青 鳉鱼富集实验的结果进行比较, 以证明TECAM只选 择性地富集自由溶解形态的HOCs, 与鱼体富集行为 类似, 是一种生物模拟采样器.

\section{1 材料与方法}

（i ）膜材料及实验生物. TECAM 膜的制备方 法及物化性质参见文献[14]. TECAM 外层为亲水相 (醋酸纤维素), 内部为疏水的脂相(三油酸甘油酯). 将 膜裁成 $4 \mathrm{~cm} \times 6 \mathrm{~cm}$ 大小, 洗净后于 Milli-Q 超纯水 (Millipore，美国)中保存备用。受试生物日本青鳞 (Oryzias latipes)由本实验室自行孵化驯养, 挑选体长 为 $3 \mathrm{~cm}$, 体重为 $0.5 \mathrm{~g}$ 左右的非产卵期成年雌性体供试. 由于青鳉的排泄物可能会影响与腐殖酸结合的 PAHs 量, 因此暴露实验前 $24 \mathrm{~h}$ 停止投食以清空其肠胃.

(ii) 微耗式采样条件的选择. 当提取的目标化 合物量小于体系中该化合物总量的 $10 \%$ 时, 可认为 测量介质不会对HOCs在水中的自由溶解态和DOC结 合态之间的平衡状态造成影响, 即 “微耗式采样”[13]. 由于本研究的实验均在静态暴露体系中进行, 为保 证膜和鱼中富集的PAHs足够被检出, 同时使溶液中 PAHs的自由溶解态浓度保持相对恒定, 暴露时需要 选择合适的条件(富集速率常数和暴露时间).

向 4 个具塞雉形瓶中各加入 $1 \mathrm{~L}$ 超纯水(实验用 水皆为 Milli- $Q$ 水, $\left.[D O C]<0.1 \mathrm{mg} \cdot \mathrm{L}^{-1}\right)$, 然后加入范 和䓛的标准溶液, 使其在水中浓度均为 $100 \mathrm{ng} \cdot \mathrm{L}^{-1}$. 静置 $24 \mathrm{~h}$ 后各放入一张 TECAM膜, 在摇床上以 0,50 , 100 和 $150 \mathrm{r} \cdot \mathrm{min}^{-1}$ 的转速进行 $1 \mathrm{~h}$ 的恒温 $\left(25^{\circ} \mathrm{C}\right)$ 萃取 实验. 实验过程中雉形瓶均密闭, 以防止溶液中 PAHs 的挥发损失. 实验设 3 个平行样.

根据上述实验结果选择膜富集实验的振荡速率 为 $75 \mathrm{r} \cdot \mathrm{min}^{-1}$, 相同条件下将膜在 $100 \mathrm{ng} \cdot \mathrm{L}^{-1} \mathrm{PAHs}$ 标准溶液中分别暴露 $1,2,3 \mathrm{~h}$. 青鳉的富集实验与膜 实验相同, 每瓶溶液中放入 2 条青鳉, 静置暴露 1,2 , $3 \mathrm{~h}$ 后取出. 同时为进一步考查实验过程中目标化合 物总的损失, 用液液萃取测定富集 $1 \mathrm{~h}$ 前后溶液中两 种 PAHs 的浓度, 比较其差值.

(iii) 富集动力学实验. 配制 5 瓶 $1 \mathrm{~L}$ 含两种 PAHs 浓度为 $100 \mathrm{ng} \cdot \mathrm{L}^{-1}$ 的溶液, 各放入一张 TECAM, $25^{\circ} \mathrm{C}$ 恒温, $75 \mathrm{r} \cdot \mathrm{min}^{-1}$ 分别富集 $1,2,3,6$ 和 $12 \mathrm{~h}$ 后取 出以计算膜的富集速率常数 $k_{\mathrm{u}}$. 青鳉的富集动力学实 验与膜实验条件相同. 将 2 条青鳉放入一瓶 PAHs 溶液 中, 静置暴露 $1,2,3,6$ 和 $12 \mathrm{~h}$ 后取出. 所有富集实验均 采取每 $1 \mathrm{~h}$ 更换一次溶液的方式, 并设 3 个平行样.

(iv) HA 对青鳞和 TECAM 富集范和䓛的影响. 将商品腐殖酸(Fluka, 瑞士)溶于 $0.01 \mathrm{~mol} \cdot \mathrm{L}^{-1}$ 的 $\mathrm{NaOH}$ 溶液中 ( pH 12), 超声溶解 $30 \mathrm{~min}$ 后搅拌 $24 \mathrm{~h}$, 用 $0.45 \mu \mathrm{m}$ 的滤膜(使用前先煮过以去除杂质)过滤除 去大颗粒, 然后用总有机碳(TOC) 分析仪测定滤液中 $\mathrm{DOC}$ 的含量 $\left(\mathrm{mg} \cdot \mathrm{L}^{-1}\right)$. 此腐殖酸储备液置于 $4^{\circ} \mathrm{C}$ 下避 光保存备用.

用腐殖酸储备液和超纯水配制DOC含量分别为 0 (超纯水空白), 1, 2, 5, 10 和 $15 \mathrm{mg} \cdot \mathrm{L}^{-1}$ 的实验溶液, 然后用 $0.1 \mathrm{~mol} \cdot \mathrm{L}^{-1}$ 的 $\mathrm{HCl}$ 和 $\mathrm{NaOH}$ 溶液调 $\mathrm{pH} 7.5 \pm 0.3$. 取上述溶液各 $1 \mathrm{~L}$ 置于雉形瓶中, 然后加入两种 PAHs标液, 使溶液中范和䓛的浓度为 $100 \mathrm{ng} \cdot \mathrm{L}^{-1}$. 放置 $4 \mathrm{~d}$ 以使 PAHs 在 HA和水相间达到分配平衡 ${ }^{[2]}$. 此过程中雉形瓶均避光密封, 以防止PAHs降解挥发 损失.

每个瓶中放入一条青鳉, $25^{\circ} \mathrm{C}$ 下静置暴露 $12 \mathrm{~h}$. TECAM 富集实验与青鳉实验一致, 每瓶溶液中放入 一张 TECAM 膜, 密封后振荡 $12 \mathrm{~h}\left(25^{\circ} \mathrm{C}, 75 \mathrm{r} \cdot \mathrm{min}^{-1}\right)$. 每组实验需更换 11 次溶液.

（v）样品预处理. 鱼样经去离子水冲洗后置于 滤纸上, 用蘸有正己烷的脱脂棉擦拭表面以除去可 能黏附的 PAHs, 去除肠子, 称重后放入研钵, 加入 5 倍质量的无水硫酸钠研磨, 然后置于通风橛中风干. 风干后的粉末在 $10 \mathrm{~mL}$ 二氯甲烷/正己烷(1:1, 体积比) 中用细胞破碎仪超声提取 $10 \mathrm{~min}$, 重复 3 次, 每次超 声完成后更换新溶剂, 最后将 $30 \mathrm{~mL}$ 提取液合并, 离 心 $10 \mathrm{~min}$ 后取上清液, 氮吹浓缩至 $1 \mathrm{~mL}$. 浓缩液在硅 胶/氧化铝复合柱上净化, 用二氯甲烷/正己烷(3:7, 体 积比)混合溶剂 $70 \mathrm{~mL}$ 淋洗. 淋洗液氮吹浓缩至 $0.5 \mathrm{~mL}$ 左右, 加入六甲基苯作为内标, 准确定容后待测.

TECAM 样取出后先用去离子水冲洗表面, 除去 可能黏附的颗粒物, 再用干净柔软的纸巾吸干表面 水分, 置于 $10 \mathrm{~mL}$ 正己烷/二氯甲烷(1:3, 体积比)溶 剂中超声提取 $10 \mathrm{~min}$, 重复 3 次, 每次均更换新溶剂, 3 次的提取液合并, 氮吹浓缩至 $0.5 \mathrm{~mL}$, 置换为正己 烷, 加入内标后准确定容待测.

(vi) GC/MS 条件及定量分析. 采用 Agilent 
6890/5973 Network 型气相色谱质谱仪进行 PAHs 分 析, 仪器配置 DB-5MS 石英毛细管柱 $(60 \mathrm{~m} \times 0.25 \mathrm{~mm}$ $\times 0.25 \mu \mathrm{m})$. 色谱条件为: 纯度 $99.999 \%$ 的氦气作载气, 无分流进样, 恒流 $1.0 \mathrm{~mL} \cdot \mathrm{min}^{-1}$; 进样口温度 $310^{\circ} \mathrm{C}$; 检测器温度 $250^{\circ} \mathrm{C}$; SIM 模式下程序升温: 初始温度 $50^{\circ} \mathrm{C}$ 保留 $2 \mathrm{~min}, 20^{\circ} \mathrm{C} \cdot \mathrm{min}^{-1}$ 升至 $200^{\circ} \mathrm{C}$ 保留 $2 \mathrm{~min}$, $5^{\circ} \mathrm{C} \cdot \mathrm{min}^{-1}$ 升至 $240^{\circ} \mathrm{C}$ 保留 $2 \mathrm{~min}, 3^{\circ} \mathrm{C} \cdot \mathrm{min}^{-1}$ 升至 $310^{\circ} \mathrm{C}$ 保留 $6 \mathrm{~min}$; 进样体积 $1 \mu \mathrm{L}$.

以六甲基苯为内标, 将两种多环芳烃混合标样通 过检索美国标准与技术研究院(NIST)质谱谱库和色谱 峰保留时间进行定性分析, 并采用内标峰面积法作六 点校正曲线以定量计算.

(vii) 质量控制与空白实验. 每批鱼样和膜样处 理时均设一个质量控制样, 加入回收率指示物気代 菲, 其回收率平均值分别为 $63 \%$ 77\%和 $70 \%$ 85\%, 最终结果均经过回收率校正. 方法空白以及 TECAM 和鱼样的全流程空白实验均未检出目标化合物.

\section{2 结果与讨论}

\section{1 微耗式采样的实现}

振荡速率对 TECAM 的富集速率常数影响较大, TECAM 对两种 PAHs 的富集速率常数均随振荡速率 的提高而增加, 萃取 $1 \mathrm{~h}$ 后最大转速 $\left(150 \mathrm{r} \cdot \mathrm{min}^{-1}\right)$ 时 膜中范和䓛的浓度分别是静置状态下的 10.4 倍和 7.9 倍. 主要原因是范和䓛从水相分配到 TECAM 中的传 质阻力与边界层厚度成正比, 而振荡能使膜周围的 边界层厚度减小, 从而提高富集速率, 因此富集实验 宜在较大的振荡速率下进行. 根据摇床的承载负荷 限制和实验的实际操作情况, 以下实验均选取转速 为 $75 \mathrm{r} \cdot \mathrm{min}^{-1}$. 图 1 中的结果表明, 在富集 1 和 $2 \mathrm{~h}$ 后, 鱼和膜 TECAM 中的 PAHs 量均小于溶液中总量的 $10 \%$. 液液萃取测得富集 $1 \mathrm{~h}$ 前后溶液中两种 PAHs 的浓度差值未超过总量的 $10 \%$. 为确保微耗式采样 的实现，所有富集实验均采取每 $1 \mathrm{~h}$ 更换一次溶液的 方式, 以保证体系维持 PAHs 在水相和腐殖酸之间的 初始平衡状态, 并补偿由于 PAHs 被富集所减少的量 以及降解、挥发和瓶壁吸附造成的损失.

\subsection{PAHs 在 TECAM 和青鳉中的富集}

当体系不含DOC且TECAM对目标化合物的富集 处在线性阶段时, 则PAHs在TECAM中的富集可简单 地用一次线性模型表示 ${ }^{[0]}$ :

$$
C_{\mathrm{TECAM}}=C_{\mathrm{w}}^{\mathrm{t}} k_{\mathrm{u}}^{\mathrm{s}} t,
$$

其中 $C_{\text {TECAM }}$ 为膜中目标化合物的含量 $\left(\mathrm{ng} \cdot \mathrm{g}^{-1}\right), C_{\mathrm{w}}$ 为 水中化合物的浓度 $\left(\mathrm{ng} \cdot \mathrm{L}^{-1}\right), k_{\mathrm{u}}$ 为富集速率常数 $\left(\mathrm{L} \cdot \mathrm{h}^{-1} \cdot \mathrm{g}^{-1}\right), t$ 为富集时间 $(\mathrm{h})$. 如图 2 所示, 在现有的实 验条件下, TECAM 对两种 PAHs 的富集均显示出很好 的线性, $R^{2}$ 值均大于 0.9. 青鲳富集实验中, 鱼中 PAHs 的浓度也随着暴露时间的增加而呈线性递增, $R^{2}$ 值大 于 0.9. 这说明在本实验的暴露条件下, 两种采样介质 对 PAHs 的富集均处于线性阶段, 速率常数 $k_{\mathrm{u}}$ 恒定.

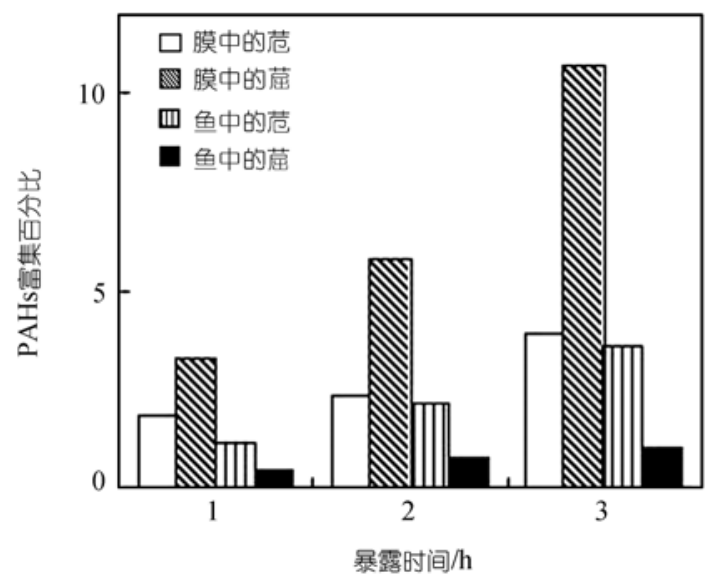

图 1 暴露不同时间后 TECAM 和鱼中 PAHs 的浓度

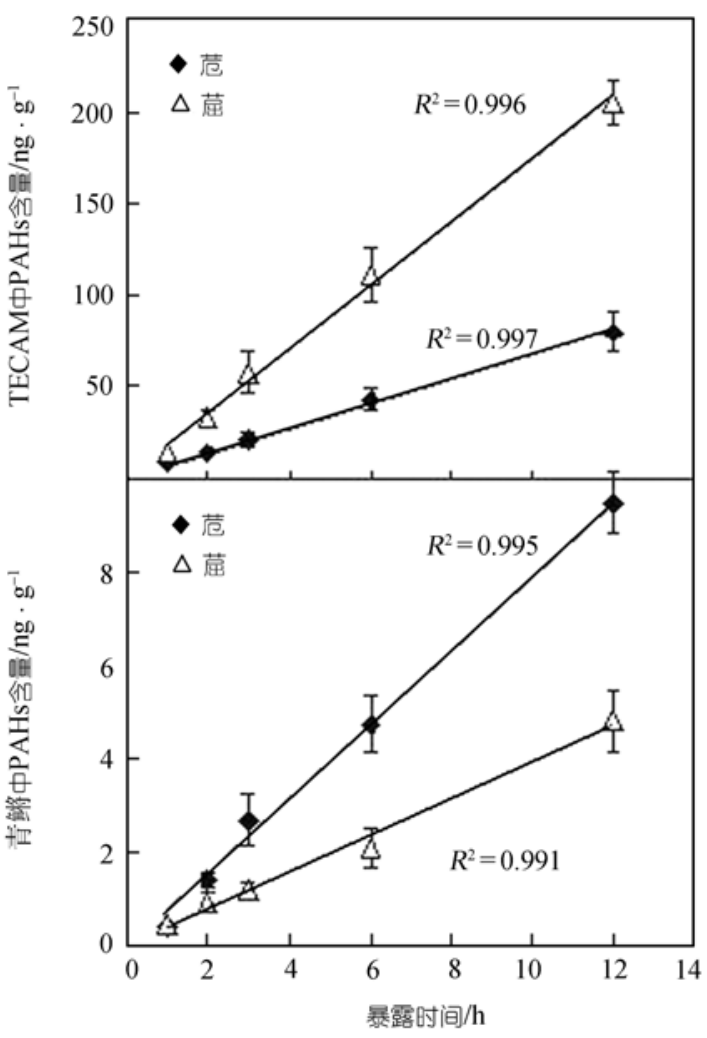

图22两种 PAHs 在 TECAM 和青鳞中的富集 


\subsection{HA 对青鳞和 TECAM 富集速率常数的影响}

$12 \mathrm{~h}$ 富集实验的结果表明, 范和䓛在青鲟体内的 浓度随着暴露溶液中 HA 浓度的增加而减少(图 3). 与不含 HA 的对照溶液相比, 在 [DOC] $=15 \mathrm{mg} \cdot \mathrm{L}^{-1}$ 的 $\mathrm{HA}$ 溶液中暴露 $12 \mathrm{~h}$ 后, 青鲟体内范的浓度从 3.43 $\mathrm{ng} \cdot \mathrm{g}^{-1}$ 减少到 $0.38 \mathrm{ng} \cdot \mathrm{g}^{-1}$, 䓛的浓度从 $8.86 \mathrm{ng} \cdot \mathrm{g}^{-1}$ 减少到 $5.90 \mathrm{ng} \cdot \mathrm{g}^{-1}$, 富集量分别减少了 $89 \%$ 和 $33 \%$. TECAM 富集实验与鱼体富集实验类似, 随着暴露溶 液中 HA 浓度的增加, TECAM 中两种 PAHs 的浓度也 呈现下降趋势(图 3), 说明 TECAM 能够模拟腐殖酸 对 PAHs 在青鲳体内的富集浓度所产生的影响.

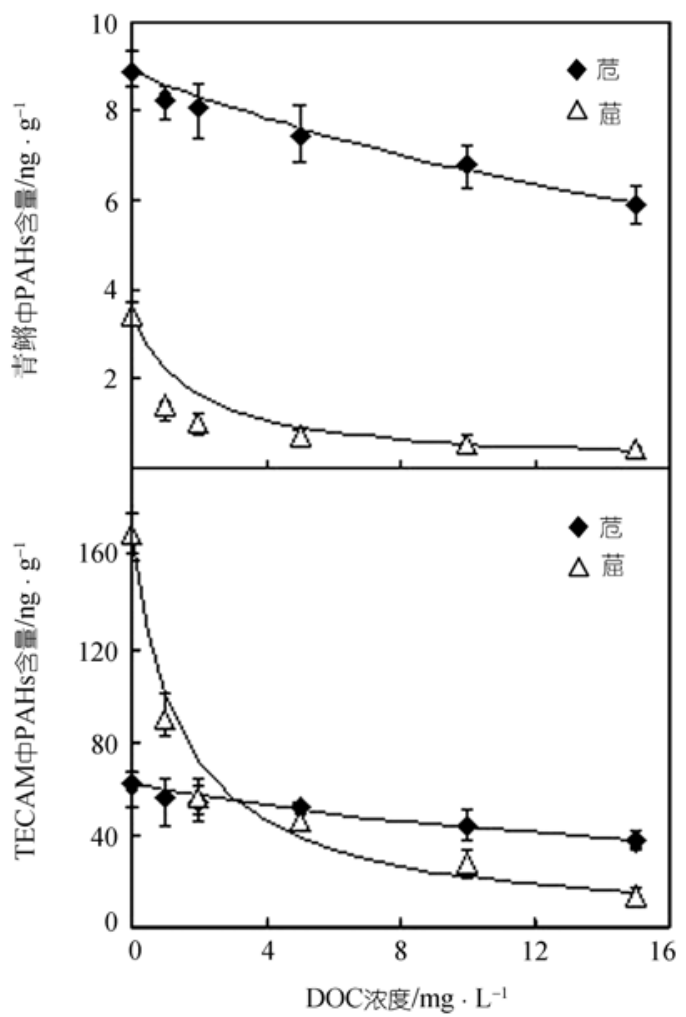

图 3 腐殖酸存在时暴露 $12 \mathrm{~h}$ 后青鳞和 TECAM 中的 PAHs 浓度

由于青鳉在暴露前已停止喂食, 实验过程中其消 化道吸收的多环芳烃不会显著影响其他器官, 且称重 前已将肠子取出, 因此可以忽略经摄食途径进入鱼体 的 PAHs 的干扰, 可认为最后所得范和䓛的浓度主要 是由鳃呼吸途径而在鱼体内富集的自由溶解态浓度, 即生物有效态浓度, 所以公式(1)中的 $k_{\mathrm{u}}$ 实际上代表了 鱼体对水中自由溶解态化合物的富集速率常数, 即

$$
k_{\mathrm{u}}=\frac{C_{\text {fish }}}{C_{\text {free }} t} \text {, }
$$

$C_{\text {fish }}$ 为鱼中的 PAHs 浓度, $C_{\text {free }}$ 为水中 PAHs 自由溶解 态浓度. 当溶液中有腐殖酸存在时, 部分 PAHs 与之 结合而使水相中的游离态浓度降低, 从而降低青鳉 的富集量. 此时, 青鳉体内的 PAHs 浓度与溶液中化 合物总浓度的关系为

$$
C_{\text {fish }}=F C_{\mathrm{w}} k_{\mathrm{u}} t,
$$

其中 $F$ 表示溶液中自由溶解态化合物占化合物总量的 比例, 可以通过下面的公式表示 $[15]$

$$
F=\frac{1}{1+K_{\mathrm{DOC}}[\mathrm{DOC}]},
$$

式中 $K_{\mathrm{DOC}}$ 是 PAHs 在 $\mathrm{HA}$ 和水相中的平衡分配系 数, [DOC]为溶解性有机碳含量. 为了描述 DOC 对青 鳞富集 PAHs 的影响, 定义 $k_{\mathrm{u}}^{\mathrm{obs}}$ 为表观富集速率常数:

$$
k_{\mathrm{u}}^{\mathrm{obs}}=k_{\mathrm{u}} F=\frac{k_{\mathrm{u}}}{1+K_{\mathrm{DOC}}[\mathrm{DOC}]}=\frac{C_{\mathrm{fish}}}{C_{\mathrm{w}} t},
$$

由于它的计算以加入腐殖酸溶液中的 PAHs 总浓度为 基础, 必然与生物实际的富集速率常数 $k_{\mathrm{u}}$ 存在偏差, 由公式(4)可得, 鱼中的 $k_{\mathrm{u}}^{\mathrm{obs}}$ 与 $k_{\mathrm{u}}$ 以及 [DOC]的关系可 以表示成以下形式:

$$
\frac{k_{\mathrm{u}}}{k_{\mathrm{u}}^{\mathrm{obs}}}=\frac{1}{F}=1+K_{\mathrm{DOC}}[\mathrm{DOC}] .
$$

相应地, 若 TECAM只富集自由溶解态的 PAHs, 其表 观富集常数也可以用以上同一模型公式表示.

由于鱼和膜的暴露时间相同, 而且溶液中 PAHs 的总浓度保持不变, 因此图 3 中两种介质富集的 PAHs 量随着腐殖酸浓度的变化也就同时反映出各自 $k_{\mathrm{u}}^{\mathrm{obs}}$ 的变化趋势. 另一方面, 由于鱼和 TECAM 各自 的富集条件不变, 腐殖酸存在时的 $k_{\mathrm{u}}$ 应与不含腐殖 酸时的数值相同. 作图 4 以验证实验中测定的 $k_{\mathrm{u}} / k_{\mathrm{u}}^{\mathrm{obs}}$ 对溶液中 [DOC]的关系, 青鳞和 TECAM 两组实验数 据都与公式(6)拟合得很好, $R^{2}$ 均大于 0.9. 说明在线 性富集阶段, 暴露溶液中腐殖酸的存在使得青鲟和 TECAM 对两种 PAHs 的表观富集常数 $k_{\mathrm{u}}{ }^{\mathrm{obs}}$ 有不同程 度的减小, 而且随着腐殖酸浓度变大, 同一化合物的 $k_{\mathrm{u}}^{\mathrm{obs}}$ 与真实的 $k_{\mathrm{u}}$ 值之间偏离得越远.

通过拟合回归，青鳞和 TECAM 两种方法测定的 范和䓛的 $\lg K_{\mathrm{DOC}}$ 值与文献报道的用其他方法测定的值 相吻合(表 1), 说明公式(6)的模型对于 TECAM也成立, 从而证明了 TECAM 能选择性富集水中自由溶解态的 PAHs, 它所表征的污染物浓度是生物有效态浓度. 此 外许多研究表明, 化合物的 $K_{\mathrm{DOC}}$ 值与其亲脂性密切相 关 $[4,10,17]$. 由于䓛的亲脂性比范强得多, 更易分配到 DOC相中, 同一浓度DOC对䓛自由态浓度的降低影 


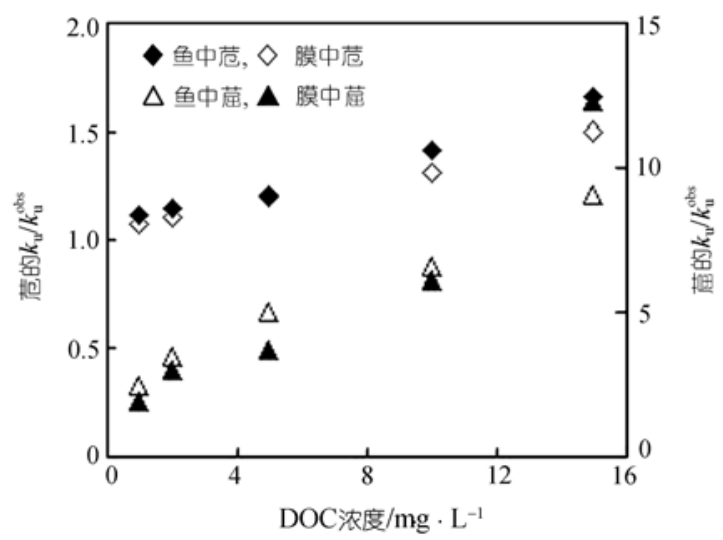

图 4 不同 DOC 浓度下膜和鱼中 $k_{\mathrm{u}}^{\mathrm{obs}}$ 与 $k_{\mathrm{u}}$ 的偏离度

表 1 用 TECAM 和青鳞富集两种方法测得的 $K_{\mathrm{DOC}}$

\begin{tabular}{cccc}
\hline PAHs & $\lg K_{\text {Doc }}{ }^{[16]}$ & $\lg K_{\text {DOC }}$ (青鳉) & $\lg K_{\text {DOC }}($ TECAM) \\
\hline 范 & 4.2 & 4.63 & 4.52 \\
䓛 & 5.3 & 5.83 & 5.76 \\
\hline
\end{tabular}

响更大. 因此在本研究的 DOC 浓度下, 尤其在较低浓 度时, 范的富集变化不会像䓛那样显著. 由图 3 可见, 两种采样介质中, 随 DOC 浓度的增加, 䓛富集浓度的 曲线变化趋势十分明显, 而范的变化趋势接近线性, 很好地验证了这一理论.

Chiou ${ }^{[18]}$ 研究发现疏水性有机污染物在三油酸甘 油酯中的累积与在鱼体脂肪内的累积类似. TECAM 采样可同时满足生物模拟采样的被动式、富集效 应 ${ }^{[19]}$ 、微耗和表征自由溶解态这几大特征, 因此可作 为一种标准器件来进行模拟生物采样, 用于测定水 中疏水性污染物的生物有效态浓度，并根据其富集 速率常数的变化来估测水体环境中DOC的存在对疏 水性有机污染物在鱼体内的富集量，即其生物有效 性的影响.

\section{3 结论}

（i ）水中存在的腐殖酸能降低青鳉对范和䓛的 表观富集常数 $k_{\mathrm{u}}^{\mathrm{obs}}$, 并导致生物体内富集浓度的降低, 而且 $k_{\mathrm{u}}^{\mathrm{obs}}$ 与真实富集速率常数 $k_{\mathrm{u}}$ 间的偏离度与暴露 溶液中的 HA 浓度有一定的正相关性 $\left(R^{2}>0.9\right)$. 说明 $\mathrm{HA}$ 的存在会减少水中 PAHs 的自由溶解态浓度, 并 降低其生物可利用性.

(ii) TECAM 富集的是水中自由溶解态的 PAHs, 可以用来测定其生物有效态浓度, 同时能够反映水 中腐殖酸的存在对青鳉富集 PAHs 的影响, 以此计算 其腐殖酸-水平衡分配常数. 因此 TECAM 可作为一 种模拟生物采样器来定量测定水环境中 DOC 对鱼体
富集 PAHs 的影响, 预测污染物的生物有效性.

\section{参考文献}

1 Wershaw R L. Model for humus in soils and sediments. Environ Sci Technol, 1993, 27(5): 814-816[DOI]

2 Akkanen J, Kukkonen J V K. Measuring the bioavailability of two hydrophobic organic compounds in the presence of dissolved organic matter. Environ Toxicol Chem, 2003, 22(3): 518-524[DOI]

3 Landrum P F, Reinhold M D, Nihart S R, et al. Predicting the bioavailability of organic xenobiotics to Pontoporeia hoyi in the presence of humic and fulvic materials and natural dissolved organic matter. Environ Toxicol Chem, 1985, 4(4): 459—467

4 Mott H V. Association of hydrophobic organic contaminants with soluble organic matter: evaluation of the database of $K_{\mathrm{doc}}$ values. Adv Environ Res, 2002, 6: 577-593[DOI]

5 Menzie C A, Potocki B B, Santodonato J. Exposure to carcinogenic PAHs in the environment. Environ Sci Technol, 1992, 26(7): 1278-1284[DOI]

6 李庆玲, 徐晓琴, 黎先春, 等. 固相微萃取-气相色谱-质谱联用 测定海水和沉积物间隙水中的痕量多环芳烃. 中国科学 B 辑: 化学, 2006, 36(3): 202-210

7 Wei C, Frank S, Weiping L, et al. Effects of dissolved organic matter on permethrin bioavailability to Daphnia species. Agric Food Chem, 2006, 54(11): 3967-3972[DOI]

8 Södergren A. Solvent-filled dialysis membranes simulate uptake of pollutants by aquatic organisms. Environ Sci Technol, 1987, 21(9): 855-863[DOI]

9 Ke R H, Xu Y P, Wang Z J, et al. Estimation of the uptake rate constants for polycyclic aromatic hydrocarbons accumulated by semipermeable membrane devices and triolein-embedded cellulose acetate membranes. Environ Sci Technol, 2006, 40(12): 3906-3911[DOI]

10 Miège $\mathrm{C}$, Ravelet $\mathrm{C}$, Croue $\mathrm{J} \mathrm{P}$, et al. Semi-permeable membrane device efficiency for sampling free soluble fraction of polycyclic aromatic hydrocarbons. Anal Chim Acta, 2005, 536(1-2): 259-266[DOI]

11 Huckins J N, Tubergen M W, Manuweera G K. Semipermeable membrane devices containing model lipid: A new approach to monitoring the bioavailability of lipophilic contaminants and estimating their bioconcentration factor. Chemosphere, 1990, 20(5): 533552 [DOI]

12 Xu Y P, Wang Z J, Ke R H, et al. Accumulation of organochlorine pesticides from water using triolein embedded cellulose acetate membranes. Environ Sci Technol, 2005, 39(4): 1152-1157[DOI]

13 Poerschmann J, Zhang Z Y, Kopinke F D, et al. Solid phase microextraction for determining the distribution of chemicals in aqueous matrices. Anal Chem, 1997, 69(4): 597-600[DOI]

14 许宜平, 吕怡兵, 李剑, 等. 新型醋酸纤维素复合膜的制备及其 基本性能研究. 高技术通讯, 2004, 14(1): 89-94

15 Anderson P F, Elsa A G, Frans J B, et al. Estimating impact of humic acid on bioavailability of hydrophobic chemicals in guppies using kinetic solid-phase extraction. Environ Toxicol Chem, 1998, 17(6): 998-1004[DOI]

16 Jeffrey N B, Barrie M P. Determination of colloidally-associated polycyclic aromatic hydrocarbons (PAHs) in fresh water using C18 solid phase extraction disks. Anal Chim Acta, 2003, 486(2): 159169[DOI]

17 霍金仙, 刘会娟, 曲久辉, 等. 三油酸甘油酯复合吸附剂去除狄 氏剂和异狄氏剂的研究. 科学通报, 2005, 50(18): 1957-1961

18 Chiou C T. Partition coefficients of organic compounds in lipid-water systems and correlations with fish bioconcentration factors. Environ Sci Technol, 1985, 19(1): 57-62[DOI]

19 柯润辉, 乔敏, 许宜平, 等. 模拟生物采样和原位鱼体生物富集 水中 PAHs 的比较. 环境科学, 2006, 27(7): 1410-1414 